\title{
From a differentiable to a real analytic perturbation theory, applications to the Kupka Smale theorems
}

\author{
H. W. BROER AND F. M. TANGERMAN \\ Department of Mathematics, University of Groningen, G.P.O.800,9700 AV Groningen, \\ The Netherlands; Department of Mathematics, Boston University, \\ 111 Cummingston Street, Boston, MA 02215, U.S.A. \\ (Received 17 April 1985 and revised 5 November 1985)
}

Abstract. Kupka-Smale like theorems are proven in the real analytic case, using existing perturbation schemes for the smooth case and the heat operator. As a consequence, a topological proof is obtained of Siegel's theorem on the generic divergence of normal form transformations.

\section{Introduction}

(a) We start by presenting some results. Recently Takens [31] renewed interest in the theory of real analytic perturbations by introducing a kind of real analytic bump function. This was used to develop a real analytic parallel of his $C^{\infty}$-perturbationscheme [30], where compactly supported bump functions occur. This led to the question whether a perturbation scheme as needed for the Kupka Smale theorems, e.g. see Kupka [13], Smale [26], Peixoto [19], Brunovksy [6], Sotomayor [27], Robinson [22] or Palis and de Melo [18], can be similarly carried over to the world of real analytic systems. We claim that they all can. Presently we shall formulate two examples of this.

In the following, $M$ shall be an $n$-dimensional real analytic manifold, endowed with a real analytic Riemannian metric. In the cases where we have to respect a volume or a symplectic structure on $M$, the metric is required to be in accordance with this structure. For details see $\S 3(\mathrm{~d})$ below.

1.1. TheOREM. Let $\mathfrak{X}^{\omega}(M)$ be the space of real analytic vector fields on $M$. Then the set $\mathrm{K}-\mathrm{S} \subseteq \mathfrak{X}^{\omega}(M)$ is residual. Here by definition $X \in \mathrm{K}-\mathrm{S}$ if:

(i) all critical elements (equilibria, closed orbits) are hyperbolic;

(ii) the stable and unstable manifolds of these critical elements are mutually transversal.

1.2. THEOREM. Let $\tau$ be a real analytic symplectic 2 -form on $M$ and let $\mathfrak{X}_{\tau}^{\omega}(M)$ be the space of real analytic vector fields on $M$ which are Hamiltonian with respect to $\tau$. Then the properties $\mathrm{H}-2$ and $\mathrm{H}-3$ define a residual subset of $\mathfrak{X}_{\tau}^{\omega}(M)$.

Remarks. (i) For a detailed description of the properties $\mathrm{H}-2$ and $\mathrm{H}-3$ we refer to $\S 4$, below. Also see Robinson [22]. H-2 concerns the fact that equilibria and closed 
orbits are elementary, while $\mathrm{H}-3$ deals with the transversality of the strong stable and unstable manifolds;

(ii) It is to be noted that also Takens [29], which deals with generic properties of Hamiltonian closed orbits expressed in terms of higher order jets, can be similarly carried over to the analytic setting.

(b) There now follows a brief outline of this paper. First, in $\S 2$, we recall some elements from the theory of real analytic systems. We include a new proof of the fact that the strong stable and unstable manifolds of critical elements are immersed real analytic manifolds. Also in $\S 2$ we specify the real analytic topology, which corresponds to local uniform convergence on complexified domains. It is of importance to us that this topology has the Baire property. Also we shall use the fact that our analytic topology is stronger than the weak Whitney- $C^{\infty}$-topology, implying that for any $k$ a $C^{k}$-open property is also open in the analytic topology.

In $\S 3$ we introduce our perturbation scheme serving to prove the analytic Kupka Smale theorems. At each of countably many stages of this scheme one has to show that a certain property $\mathfrak{B}$, depending on the stage under consideration, is open and dense in the analytic topology, knowing that it is $C^{k}$-open and -dense for some $k \geq 1$. From the above we conclude that the openness is no problem. This property $\mathfrak{B}$ always can be expressed in terms of transversality in bundles of finite jets. The classical proofs of the smooth Kupka Smale theorems, see e.g. [13], [26], [19], [6], [27], [22] provide us with smooth transversal families, which are, or can be used to obtain the corresponding open- and dense-ness. Now our method applies the heat operator (cf. e.g. Berger et al. [2], Cheval [7]) to these families, yielding nearby analytic families which then also are transversal. (In the case where $M=\mathbb{P}^{n}$ this amounts to convolution with a Gaussian kernel.) From this we conclude that our property $\mathfrak{B}$ also is dense in the analytic topology.

So unlike Takens [31] we do not construct completely new analytic perturbations, but turn smooth perturbations into analytic ones by the heat operator. Similar methods to obtain analytic approximations have been used by e.g. Whitney [34], Bochner [4], Morrey [15] and Grauert [10], though such approximations by convolutions go back to Weierstrass [33].

In $\$ 4$ we display to some detail how our approach applies to the $C^{1}$-proof of the Kupka-Smale theorem, as presented in Palis and de Melo [18], thereby giving a complete proof of theorem 1.1 .

Heuristically speaking the main problem with any such an infinite perturbation scheme seems to be that, since analytic perturbations do not have compact support, at each stage of it one has to make sure that one doesn't ruin achievements accomplished at earlier stages of the scheme. This problem, however, is not felt, since at each stage we work in an open and dense set of our analytic topology, in the end ensuring convergence of the whole process using the Baire property.

Remark. We approach the 'Kupka-Smale' properties $\mathfrak{B}$ under consideration from the rather technical viewpoint of transversality. As an example of a generic property which is not of this type, we mention the one the closing lemma is concerned with, 
cf. Pugh [20], [21]. For a fundamental discussion of generic properties which are or are not 'Kupka-Smale' and their behaviour with respect to the addition of extra parameters, see Takens [32].

(c) Our methods also provide a more topological proof of Siegel [25]. In [25] analytic Hamiltonian systems are considered near an elliptic equilibrium point without resonances. At this point the system has a Birkhoff normal form. It is shown that generically the formal canonical conjugacy from the system to its normal form, has zero radius of convergence.

Let us briefly describe the topology in question. Siegel considers all germs of real analytic Hamilton functions having a fixed 2-jet $\sum_{j=1}^{n}\left(\beta_{j} / 2\right)\left(p_{j}^{2}+q_{j}^{2}\right), n$ being the number of degrees of freedom and where the characteristic frequencies $\beta_{1}, \beta_{2}, \ldots, \beta_{n}$ are all positive and without resonances, see above. This space of germs is endowed with the topology of convergence of corresponding Taylorcoefficients, after an appropriate normalization which puts all of these coefficients in the complex unit disk. Our topology of uniform convergence on complexified domains is compatible with this, by the Cauchy integral formula.

Siegel's proof is of a rather analytic nature whereas our perturbation methods give rise to a more topological proof. We now give a brief description of how it runs. One step is to show that generically closed orbits with an a priori bounded period are isolated in each energy level. Here we meet a 'Kupka-Smale' property contained in property $\mathrm{H}-2$, cf. theorem 1.2. The other step of our proof is to ensure that closed orbits occur in every neighbourhood of the elliptic point. (The a priori bound has to increase to infinity as this neighbourhood collapses.)

To be more precise we approximate a $C^{\infty}$ Kupka Smale perturbation scheme, where a completely integrable system is perturbed flatly (i.e. with an identically vanishing Taylor series in the elliptic point) in the space of real analytic systems. Compare Takens [30], [31] where a similar programme is carried out. Also see below.

Since analytic perturbations do not have compact support it is an extra problem how to keep the characteristic frequencies of the elliptic equilibrium constant. We can overcome this difficulty by changing the above set-up slightly. In fact we introduce $n$ 'detuning' parameters, such that the frequencies 'vary with these parameters'. For $n$-parameter families of Hamiltonian systems it is a 'Kupka-Smale' property that closed orbits with an a priori bounded period are isolated in each energy level and for each value of the parameters, compare above. Now, after a small perturbation of the family one can find a unique parameter value for which the characteristic frequencies are $\beta_{1}, \beta_{2}, \ldots, \beta_{n}$. From here the proof is straightforward.

Remarks. (i) It is noted that for a special case similar arguments can be drawn from Moser [16] or Zehnder [35]. In [16, thm. 3], it is stated that for a generic area preserving map of the plane, in any neighbourhood of an elliptic fixed point periodic points of arbitrarily high period occur, which for bounded period are isolated. Many of these periodic points are hyperbolic. Now [35] proves moreover that generically the invariant manifolds of such a hyperbolic point meet transversally, so giving rise 
to a (transverse) homoclinic point. Moser and Zehnder both use the same topology on the space of analytic germs. This topology is finer than Siegel's.

Disregarding the matter of different toplogies (for a remark on this cf. $\S 2$ below), we conclude that [16, thm. 3] is contained in our result. Also we conjecture that our analytic approximation of a $C^{\infty}$ perturbation scheme may provide a new and simplified proof of [35]. Compare Robinson [23].

(ii) More generally speaking there are cases where formal normal form theorems, if they were not only formal but valid on a whole neighbourhood, would imply a certain symmetry. In some situations this symmetry is in conflict with transversality, resp. genericity. In the analytic case this leads to divergence of the normalizing transformation, while in the $C^{\infty}$-case we then have to do with a flat dynamical phenomenon. This means that the phenomenon in question (such as homoclinic orbits in every neighbourhood) can be cancelled by a flat perturbation. Examples of this are Takens [30] and Broer \& Vegter [5]. In the same spirit as [30], [31] it is also possible to give an analytic parallel of [5].

(d) After this paper was written Clark Robinson brought to our attention that Leslie [14] proved a real analytic Kupka Smale theorem for diffeomorphisms on a compact manifold $M$, to be denoted by $\operatorname{Diff}^{\omega}(M)$. It turns out that the Silva-Van Hove topology on the group $\operatorname{Diff}^{\omega}(M)$, which Leslie uses for his purpose, is coarser than the one we use, though it is finer than the Whitney $C^{\infty}$-topology. For a remark on the subject of different topologies again we refer to $\S 2$ below. The main idea now is to endow this group with the structure of $C^{\infty}$-Lie-group, for which an implicit function theorem can be proven, adequate for transversality theory. In this way the 'classical' transversality theory, cf. e.g. Hirsch [12], can be mimicked. Important moreover is to have some analogue of Sard's lemma, serving to prove denseness, see above. Also it is shown by an analytic imbedding of $M$ in Euclidean space, that there exist sufficiently many analytic perturbations of the identity map of $M$ : analytic vector fields on the ambient space are projected on the manifold $M$ and integrated subsequently.

Clearly this method is quite different from ours. Moreover ours seems to have some advantage in that it has more general applications. To start with, the compactness seems to be an indispensable ingredient of Leslie [14], while our method does not need it. Also Leslie constructs perturbations using an ambient Euclidean space, while we use local perturbations and the metric of $M$, which makes it easier to respect certain structures (such as volume forms, symplectic forms) in the considerations.

(e) Acknowledgements. The authors would like to thank Floris Takens for suggesting this problem to them and also for encouragement and valuable advice. Also they are very indebted to Mark Kon for his enthusiastic help concerning the heat equation. Gratitude moreover is due to Clark Robinson for encouragement and for pointing out the relation with Leslie's work. Finally the first author wishes to thank Boston University for its hospitality during the period when this paper was finished. 


\section{Real analytic systems}

In this section we confine ourselves to the case of $\mathbb{R}^{n}$, but the considerations can be directly carried over to the general case of real analytic manifolds (e.g. use the fact that any real analytic manifold with a countable base, can be analytically imbedded in Euclidean space, cf. Grauert [10]).

We begin with some elementary definitions.

A function $f: \mathbb{R}^{n} \rightarrow \mathbb{R}$ is called real analytic $\left(f \in C^{\omega}\left(\mathbb{R}^{n}\right)\right)$ if $f$ has a complex analytic (= holomorphic) extension to an open neighbourhood of $\mathbb{R}^{n}$ in $\mathbb{C}^{n}$. A vector field $X=\sum_{i=1}^{n} f_{i} \partial / \partial x_{i}$ then is called real analytic $\left(X \in \mathfrak{X}^{\omega}\left(\mathbb{R}^{n}\right)\right)$ if all its component functions $f_{i}$ are. A similar definition goes for maps $\mathbb{R}^{n} \rightarrow \mathbb{R}^{m}$.

The set $C^{\omega}\left(\mathbb{R}^{n}\right)$ obtains a topology as follows (inducing topologies for vector fields and maps), cf. Takens [31]:

(i) A basis of neighbourhoods for the zero function is given by considering for all compact sets $K \subseteq \mathbb{R}^{n}$ and for all choices of positive real numbers $\sigma$ and $\varepsilon$ the set $U(K, \sigma, \varepsilon) \subseteq C^{\omega}\left(\mathbb{R}^{n}\right)$, which precisely contains the functions $f \in C^{\omega}\left(\mathbb{R}^{n}\right)$ that on $K$ have a holomorphic extension to the strip

$$
K+\sigma:=\left\{z \in \mathbb{C}^{n}|\exists x \in K:| z-x \mid \leq \sigma\right\},
$$

such that for $|f|_{K, \sigma}:=\sup _{K+\sigma}|f(z)|$ one has $|f|_{K, \sigma}<\varepsilon$;

(ii) A basis of neighbourhoods for any other function in $C^{\omega}\left(\mathbb{R}^{n}\right)$ is obtained by translation of the basis under (i).

Remarks. (i) Our definition makes $C^{\omega}\left(\mathbb{R}^{n}\right)$ a Baire space, while for $0 \leq r \leq \infty$ the inclusion $C^{\omega}\left(\mathbb{R}^{n}\right) \rightarrow C^{r}\left(\mathbb{R}^{n}\right)$ is continuous in the weak Whitney $C^{r}$-topology. See Takens [31].

(ii) Although by (ii) of the above definition addition is continuous in $C^{\omega}\left(\mathbb{R}^{n}\right)$, we do not obtain a topological vector space, since scalar multiplication is not continuous. As an example consider $f \in C^{\omega}\left(\mathbb{R}^{n}\right)$ which is not entire, i.e. $f$ does not have a holomorphic extension to all of $\mathbb{C}^{n}$, and consider the arc $\lambda \in \mathbb{R} \rightarrow \lambda f \in C^{\omega}\left(\mathbb{R}^{n}\right)$. This arc is not continuous, in fact it inherits the discrete topology.

(iii) If $E\left(\mathbb{R}^{n}\right)$ denotes the set of entire real analytic functions on $\mathbb{R}^{n}$, i.e. the ones admitting a holomorphic extension to all of $\mathbb{C}^{n}$, then $E\left(\mathbb{R}^{n}\right)$ from the above definition inherits the compact-open topology, which makes it a Fréchet space. All perturbations to be carried out later on will be based on vector fields with entire component functions. For real analytic manifolds a similar remark holds. The important thing is that the complex domains of definition, during the infinite perturbation scheme, do not shrink to zero in the complex direction. In this way examples like

$$
f_{k}(x)=e^{-k / 1+k|x|^{2}}, \quad k \in \mathbb{N},
$$

which for $k \rightarrow \infty$ tends to a $C^{\infty}$-function which is not real analytic, are excluded. We are indebted to M. R. Herman and to R. Douady for helpful discussions on this subject.

(iv) From remark (ii), above, it is clear that our topology is very fine. However, if we take any real analytic topology $T$ which is coarser than ours, but - like ours, see remark (i) - finer than a Whitney $C^{r}$-topology $r \geq 1$ then the following holds. If $\mathfrak{B}$ is a 'Kupka Smale' property, then $\mathfrak{B}$ is open and dense in $T$. In fact $\mathfrak{B}$ is open 
and dense in the $C^{r}$-topology and therefore open both in $T$ and in our topology, since both are finer, see above. Moreover our results yield that $\mathfrak{B}$ is also dense in our topology which implies that $\mathfrak{B}$ is dense in $T$, since $T$ is coarser than our topology. We thank J. C. Yoccoz for discussions leading to this remark.

Now we return to vector fields. Observe that by the real analytic inverse- and implicit function theorems, compare e.g. Narasimham [17], one has that for a real analytic vector field $X$, away from its equilibrium points, there exist local real analytic flowboxes (i.e. local analytic conjugacies to the standard vector field $\partial / \partial t$ ); also a Poincaré map of $X$, defined between real analytic transverse sections, is real analytic. Furthermore we have:

2.2. Theorem. Let $X \in \mathfrak{X}^{\omega}\left(\mathbb{R}^{n}\right)$ and let $p$ be an equilibrium point of $X$. Then the strong stable and unstable manifolds of $p$, resp. to be denoted by $W^{s}(p)$ and $W^{u}(p)$, are real analytic, immersed submanifolds of $\mathbb{R}^{n}$.

Proof. Extend $X$ to a neighbourhood of $\mathbb{R}^{n}$ in $\mathbb{C}^{n}=\mathbb{R}^{n} \oplus i \mathbb{R}^{n}$. This extension again has $p$ as an equilibrium point. Note that if $\operatorname{Re} X$ in $p$ has the linear part $A$, then so has $\operatorname{Im} X$. Let us split $T_{p} \mathbb{C}^{n}=\mathbb{C}^{n}=\tilde{E}^{c} \oplus \tilde{E}^{s} \oplus \tilde{E}^{u}$, being resp. the stable-, the centreand the unstable directions of $X$ at the point $p$.

It is known that the time $t$ map $X_{t}$ of $X$, as far as defined, extends to a holomorphic transformation. Now let $\tilde{W}^{s}(p)$ and $\tilde{W}^{u}(p)$ denote the strong stable and unstable manifolds of $p$ in $\mathbb{C}^{n} \cong \mathbb{R}^{2 n}$. They are of class $C^{\infty}$ and, by unicity, extensions of $W^{s}(p)$ and $W^{u}(p)$. We shall prove that these extensions are complex analytic manifolds, so showing that our theorem holds.

To this end recall that a $C^{\infty}$-manifold $M \subseteq \mathbb{C}^{n}$ is complex analytic as soon as, in each point $x \in M$, the tangent space $T_{x} M$ is a complex vector space, i.e. that for all $v \in T_{x} M$ also $i v \in T_{x} M$. Now for $M=\tilde{W}^{s}(p)$ (resp. $\tilde{W}^{u}(p)$ ) we already know that $T_{p} M=\tilde{E}^{s}$ (resp. $\tilde{E}^{u}$ ) is a complex vector space, since these tangent spaces are invariant manifolds of the linear part of $X$ at $p$. By the same argument $\tilde{E}^{c}$ is a complex vector space.

From now on we restrict our attention to $\tilde{W}^{s}(p)$ (everything is similar for $\tilde{W}^{u}(p)$ ). Let $D$ be a small disc around $p$ in $\mathbb{C}^{n}$. Also let $\tilde{E}_{0}^{s}$ and $\tilde{W}_{0}^{s}(p)$ denote the intersections of $\tilde{E}^{s}$ (resp. $\tilde{W}^{s}(p)$ ) with $D$. Next, for $t<0$, we define

$$
\tilde{E}_{t}^{s}:=\left\{\tilde{X}_{t}(q) \mid q \in \tilde{E}^{s} \text { and for } t \leq \tau \leq 0: \tilde{X}_{\tau}(q) \in D\right\} .
$$

From the above it follows that $\tilde{E}_{t}^{s}$ is a holomorphic manifold. By the stable manifold theorem, cf. Hartman [11, Ch. IX theorem 6.1.] we know that $\tilde{E}_{t}^{s} \rightarrow \tilde{W}_{0}^{s}(p)$, as $t \rightarrow-\infty$, in the $C^{1}$-topology. From this one directly concludes that also $\tilde{W}_{0}^{s}(p)$ is holomorphic. In fact take any $x \in \tilde{W}_{0}^{s}(p)$ and a sequence $\left\{x_{k}\right\}_{k=1}^{\infty}$ with $x_{k} \in E_{t_{k}}^{s}, x_{k} \rightarrow x, t_{k} \rightarrow-\infty$. Notice that $T_{x_{k}} \tilde{E}_{t_{k}}^{s} \rightarrow T_{x} \tilde{W}_{0}^{s}(p)$. Now, by the analyticity of the $\tilde{E}_{t_{k}}^{s}$ the $T_{x_{k}} \tilde{E}_{t_{k}}^{s}$ are complex vector spaces, i.e. closed for scalar multiplication with $i$. Clearly this carries over to the limit $T_{x} \tilde{W}_{0}^{s}(p)$. Finally saturation by the analytic flow $X_{t}$ shows that all of $\tilde{W}^{s}(p)$ is analytic.

Remarks. (i) Our proof of theorem 2.2 uses the stable and unstable manifold theory for $C^{\infty}$ vector fields, see e.g. Hartman [11], and derives analyticity in a rather 
geometric way. A different approach uses a direct contraction argument in the world of holomorphic systems, compare e.g. Chow and Hale [8]. In fact this is also the classical result. Hartman [11] indicates it in his historical notes and Coddington and Levinson [9] prove it;

(ii) It is well known that, even in this analytic case, centre manifolds are in general not $C^{\infty}$, cf. e.g. Van Strien [28];

(iii) A complete analogue of theorem 2.2 holds for periodic points of real analytic diffeomorphisms.

Finally let us consider the strong stable and unstable manifolds of closed orbits in real analytic systems. A direct approach as above does not apply here, because of the following difficulty. Although a hyperbolic equilibrium point after complex extension remains hyperbolic this is not the case for a normally hyperbolic closed orbit. This is due to the fact that the time $t$ map $X_{t}$ of our vector field $X$ extends to a holomorphic transformation. Now if $v$ is a vector tangent to the closed orbit, then $i v$ is transversal to it. Observe that both $v$ and $i v$ are eigenvectors for the derivative of $X_{t}$ corresponding to the eigenvalue 1 . This means that the Poincare return map of the complexified system always has an eigenvalue 1 . Nevertheless we have

2.3. TheOREM. Let $X \in \mathfrak{X}^{\omega}\left(\mathbb{R}^{n}\right)$ and let $\gamma$ be a closed orbit of $X$. Then the strong stable and unstable manifolds of $\gamma$, to be denoted by resp. $W^{s}(\gamma)$ and $W^{u}(\gamma)$ are real analytic, immersed submanifolds of $\mathbb{R}^{n}$.

Proof. Let $\Sigma \subseteq \mathbb{R}^{n}$ be a real analytic Poincaré section of $\gamma$ and $P: \Sigma \rightarrow \Sigma$ the corresponding return map, which has $\gamma \cap \Sigma$ as a fixed point. Using remark (ii) to theorem 2.2 we find that $W^{s}(\gamma) \cap \Sigma$ and $W^{u}(\gamma) \cap \Sigma$ are real analytic submanifolds of $\Sigma$. Then choosing a local analytic flowbox for $X$ which contains $\Sigma$, gives us real analytic charts for the local invariant manifolds $W_{0}^{s}(\gamma)$ and $W_{0}^{u}(\gamma)$, cf. the proof of theorem 2.2. Finally saturation by the analytic flow $X_{t}$ shows that $W^{s}(\gamma)$ and $W^{u}(\gamma)$ are real analytic.

Remark. If $X$ is a real analytic Hamiltonian vector field and $\gamma$ a closed orbit of $X$, then its real analytic invariant manifolds $W^{s}(\gamma)$ and $W^{u}(\gamma)$ are contained in one energy level. So both $\gamma$ and its invariant manifolds are parametrized by the energy. It is easy to see that if $\gamma$ is 1-elementary then the families of invariant manifolds one so obtains 'near $\gamma$ ', depend in an analytic way on the energy.

\section{Perturbations}

In this section we introduce suitable real analytic perturbations and build up a perturbation scheme for the purpose of proving the Kupka Smale theorems; for more details on this cf. $\S 4$ below. We start our considerations on $\mathbb{R}^{n}$, in relevant cases using the standard volume or symplectic structure. In part (d), below, we turn to the general case of real analytic manifolds.

(a) On $\mathbb{R}^{n}$ consider the Gaussian convolution kernel

$$
E(y, t):=(4 \pi t)^{-n / 2} e^{-\|y\|^{2} / 4 t} .
$$


Notice that for $t>0$ the function $E(\cdot, t)$ is real analytic, having a holomorphic extension to all of $\mathbb{C}^{n}$. As a distribution $E(\cdot, t)$ for $t \downarrow 0$ converges to the Dirac measure at the point $0 \in \mathbb{R}^{n}$. It is known, cf. Schwartz [24], that $E$ is a so called fundamental solution of the heat equation.

In the following we write $E_{t}:=E(\cdot, t)$. Also $K \subseteq \mathbb{R}^{n}$ will be a compact set and $\sigma$ a positive real number. Recall from $\S 2$ that $K+\sigma \subseteq \mathbb{C}^{n}$ is the strip of width $\sigma$ around $K,|\cdot|_{K, \sigma}$ denoting the supremum norm on it. Moreover for $k=0,1,2, \ldots$ the $C^{k}$-norm on $\mathbb{R}^{n}$ is denoted by $|\cdot|_{k}$. We now have

3.1. Perturbation lemma. Let $\phi \in C^{\infty}\left(\mathbb{R}^{n}\right)$ have compact support. Then:

(i) For $t>0$ the convolution $E_{t} * \phi$ is well defined, extending holomorphically over $\mathbb{C}^{n}$

(ii) In the (weak) Whitney $C^{\infty}$-topology $E_{t} * \phi \rightarrow \phi$ as $t \downarrow 0$;

(iii) In the analytic topology $\left|E_{1} * \phi\right|_{K, \sigma} \leq e^{\sigma^{2} / 4 t}|\phi|_{0}$.

Proof. Recall that $\left(E_{t} * \phi\right)(x)=(4 \pi t)^{-n / 2} \int_{\mathbb{R}^{n}} \phi(y) e^{-(1 / 4 t)\|x-y\|^{2}} d y$.

(i)-(ii) Cf. Whitney [34]. Clearly the above formula is well defined for all $x \in \mathbb{C}^{n}$ and, since it is independent of $\bar{x}$, it gives a holomorphic function. Next we shall prove that for $k=0,1,2, \ldots$

$$
\left|E_{t} * \phi-\phi\right|_{k}=O\left(t^{1 / 4}\right)|\phi|_{k+1}
$$

uniformly in $k$. Clearly this is sufficient for our assertion. Notice that since

$$
\frac{\partial}{\partial x_{j}}\left(E_{t} * \phi\right)=E_{t} * \frac{\partial \phi}{\partial x_{j}}
$$

etc., it is enough to check $(*)$ only for $k=0$.

In order to do this we write

$$
\left(E_{t} * \phi-\phi\right)(x)=(4 \pi t)^{-n / 2} \int_{\mathbb{R}^{n}}\{\phi(x-y)-\phi(x)\} e^{-\|y\|^{2} / 4 t} d y,
$$

and for some $\varepsilon>0$, to be specified below, split

$$
\begin{aligned}
\left|E_{t} * \phi-\phi\right|_{0} \leq & |\phi|_{1}(4 \pi t)^{-n / 2} \int_{\|y\| \leq \varepsilon}\|y\| e^{-\|y\|^{2} / 4 t} d y \\
& +2|\phi|_{0}(4 \pi t)^{-n / 2} \int_{\|y\|>\varepsilon} e^{-\|y\|^{2} / 4 t} d y \\
\leq & |\phi|_{1}(\varepsilon+2 P(t, \varepsilon)) .
\end{aligned}
$$

Here the function $P(t, \varepsilon)$ can be further estimated using the following arguments from probability theory, compare for example Billingsley [3]. Notice that $P(t, \varepsilon)$ is the probability that $\sum_{j=1}^{n} Y_{j}^{2}>\varepsilon^{2}$, where the $Y_{j}(1 \leq j \leq n)$ are independent random variables, all having the same Gaussian distribution with mean 0 and variance $2 t$. Putting $Z:=(1 / 2 t) \sum_{j=1}^{n} Y_{j}^{2}$ it is well known that $Z$ has the $\chi_{n}^{2}$-distribution, the mean and variance of which are resp. $n$ and $2 n$. So $P(t, \varepsilon)=\operatorname{Prob}\left(Z<\varepsilon^{2} / 2 t\right)$, which for $\varepsilon^{2} / 2 t>n$ can be estimated by Chebychev's inequality. In fact for such a choice of $\varepsilon$ we have

$$
P(t, \varepsilon) \leq \frac{8 n t^{2}}{\left(\varepsilon^{2}-2 n t\right)^{2}} .
$$


Now assuming that $t<1 / 2 n$ and more specifically choosing $\varepsilon:=(2 n t)^{1 / 4}$, we find directly our estimate $(*)$.

(iii) Put $x=\xi+i \eta\left(\xi, \eta \in \mathbb{R}^{n}\right)$, then

$$
\begin{aligned}
\left(E_{t} * \phi\right)(x) & =(4 \pi t)^{-n / 2} \int_{\mathbb{R}^{n}} \phi(y) e^{-(1 / 4 t) \sum_{j=1}^{n}\left(\xi_{j}+i \eta_{j}-y_{j}\right)^{2}} d y \\
& =(4 \pi t)^{-n / 2} e^{(1 / 4 t)\|\eta\|^{2}} \int_{\mathbb{R}^{n}} e^{-(1 / 4 t)\left\{\|\xi-y\|^{2}-2 i \sum_{j=1}^{n} \eta_{j}\left(\xi_{j}-y_{j}\right)\right\}} d y .
\end{aligned}
$$

Hence

$$
\begin{aligned}
\left|E_{t} * \phi\right|_{K, \sigma} & \leq e^{\sigma^{2} / 4 t}(4 \pi t)^{-n / 2} \int_{\mathbb{R}^{n}}|\phi(y)| e^{-\|\xi-y\|^{2} / 4 t} d y \\
& \leq\left.\left. e^{\sigma^{2} / 4 t}\right|_{\phi}\right|_{0}
\end{aligned}
$$

(b) With the help of lemma 3.1 we now indicate how to construct real analytic perturbations of a desired type, given compact support perturbations of that same type.

(i) If $X=\sum_{j=1}^{n} \phi_{j} \partial / \partial x_{j}$ is a vector field on $\mathbb{R}^{n}$ having compact support, then

$$
E_{t} * X:=\sum_{j=1}^{n}\left(E_{t} * \phi_{j}\right) \frac{\partial}{\partial x_{j}} \quad \text { for each } t>0
$$

is a real analytic vector field on $\mathbb{R}^{n}$, which for $t$ small is close to $X$ in the $C^{\infty}$-topology. Similarly the time-1 map $\left(E_{t} * X\right)_{1}$ is close to the time-1 map $X_{1}$ of $X$. (Diffeomorphisms of $\mathbb{R}^{n}$ will be perturbed by transformations close to the identity, which can all be taken of this form.)

(ii) Since for the divergence one has $\operatorname{div}\left(E_{t} * X\right)=E_{t} *(\operatorname{div} X)$, it follows that if $X$ has divergence zero then also $E_{t} * X$ has divergence zero. Now, taking the time-1 map $\left(E_{t} * X\right)_{1}$ gives a diffeomorphism preserving the standard volume of $\mathbb{R}^{n}$.

(iii) Let $n=2 m$ and let $\tau=\sum_{j=1}^{m} d x_{j} \wedge d y_{j}$ be the standard symplectic 2-form on $\mathbb{R}^{2 m}$. If a vector field $X$ is Hamiltonian with respect to $\tau$ then an energy function $H$ exists with $\iota_{X} \tau=d H$. It directly follows that $\iota_{E_{t} * X} \tau=d\left(E_{t} * H\right)$, implying that $E_{t} * X$ again is Hamiltonian with respect to $\tau$. Again, similar to the above, the time-1 map $\left(E_{t} * X\right)_{1}$ yields a symplectic transformation of $\mathbb{R}^{2 m}$, etc. etc.

At the end of this section, using the heat equation, we shall meet a coordinate free approach to these problems, particularly those concerning the preservation of a volume- or a symplectic structure.

Now let $X$ be a real analytic vector field on $\mathbb{R}^{n}$. Also let $X^{\mu}$ be a $C^{\infty}$-unfolding of $X=X^{0}$, where $\mu$ varies over some $s$-dimensional parameter space and where for all $\mu$ the difference $X-X^{\mu}$ has support in a fixed compact set. Then define $\tilde{X}^{\mu, t}:=X+E_{t} *\left(X^{\mu}-X\right)$. Note that $\tilde{X}^{\mu, t}$, for fixed $t>0$, is a $C^{\infty}$-unfolding of $X=\tilde{X}^{0, t}$, where now for each value of $\mu$ the vector field $\tilde{X}^{\mu, t}$ is real analytic. Given $K \subseteq \mathbb{R}^{n}$ as a compact set, a positive integer $k$ and a positive real number $\sigma$, we let $|\cdot|_{K, k}$ denote the $C^{k}$-norm on $K$ and $|\cdot|_{K, \sigma}$ the supremum norm on the complex strip $K+\sigma \subseteq \mathbb{C}^{n}$, see above. Also, as before, $|\cdot|_{k}$ denotes the $C^{k}$-norm in the case of compact support. We then have, as a direct corollary to lemma 3.1 


\subsection{Perturbation lemma:}

$$
\begin{gathered}
\left|\tilde{X}^{\mu, t}-X^{\mu}\right|_{K, k}=O\left(t^{\frac{1}{4}}\right)\left|X^{\mu}-X\right|_{k+1} ; \\
\left|\tilde{X}^{\mu, t}-X\right|_{K, \sigma} \leq e^{\sigma^{2} / 4 t}\left|X^{\mu}-X\right|_{0} .
\end{gathered}
$$

Proof. $\tilde{X}^{\mu, t}-X^{\mu}=\left(X^{\mu}-X\right)+E_{t} *\left(X^{\mu}-X\right)$. Now apply lemma 3.1 and its proof, in particular, formula $(*)$.

Remark. Evidently $\tilde{X}^{\mu, t}$ need not converge to $X^{\mu}$ in the analytic topology! Moreover notice that the constant $O\left(t^{\frac{1}{4}}\right)$ is completely determined by the Gaussian kernel, so does not depend on $K$ or on supp $\left(X^{\mu}-X\right)$.

(c) The perturbation scheme. The Kupka Smale theorems and other, similar, genericity theorems, e.g. see Kupka [13], Smale [26], Peixoto [19], Robinson [22], Brunovksy [6], Sotomayor [27], Takens [29], Palis and de Melo [18] and others, all can be proven using an inductive perturbation scheme. At each step of such a scheme one works in an open and dense set corresponding to the preceding part of the scheme and one has to show that the property belonging to the present step only gives rise to new open and dense restrictions. The fact that one has to do with a Baire space then ensures that such a countable process 'converges', yielding a dense residual set in which all these properties hold. The nature of the properties at hand, and of the corresponding perturbation arguments will be discussed below. One, for example, has to think of hyperbolicity of all equilibrium points in a given compact disk. In $\S 4$, below, we will present more examples.

Remark. In the perturbation scheme several parameters, like the radius of the compact disk to which the attention is restricted or the maximum period of the closed orbits one considers, etc., play a rôle. During the induction process some of these have to increase to infinity. For more details in a specific example see $\$ 4(\mathrm{a})$, below. If one chooses a suitable diagonal procedure one can ensure that indeed at each step one deals with an open and dense set.

Now let us specify what is meant by a 'Kupka Smale' property. Suppose, for simplicity, that our concern is with vector fields $X$ varying over the open set to which we restrict from a given point of our scheme. We shall consider a property $\mathfrak{B}$ which holds for a subset of these vector fields. $\mathfrak{P}$ is referred to as a 'Kupka Smale' property if the following holds. With $X$ a smooth map $f_{X}: M \rightarrow N$ is associated, where $M$ and $N$ are finite dimensional manifolds, $M$ being compact, and where in $N$ a certain (stratified) manifold $S$ is given. Here it is important that $M, N$ and $S$ are independent of $X$, while the map $f_{X}$ depends smoothly on $X$. Now suppose that we arranged our choice of $f_{X}, M, N$ and $S$ in such a way that $X$ has property $\mathfrak{B}$ if and only if $f_{X} \nwarrow S$. Also suppose that a smooth $s$-parameter unfolding $X^{\mu}$ of $X=X^{0}$ is given such that for $F: M \times \mathbb{R}^{s} \rightarrow N$, defined by $F(\cdot, \mu):=f_{X^{\mu}}$, one has $F \aleph_{M \times\{0\}} S$. In our applications it will be no loss of generality to assume that for all $\mu$ the perturbation $X^{\mu}-X$ is of class $C^{\infty}$ and has its support in some fixed compact set, see e.g. [13], [26], [19], [6], [27], [22], [29]. Then, according to the Thom transversality principle, cf. e.g. Hirsch [12], the set

$$
\left\{\mu \in \mathbb{R}^{s} \mid X^{\mu} \text { has property } \mathfrak{B}\right\}=\left\{\mu \in \mathbb{R}^{s} \mid f_{X^{\mu}} \nwarrow S\right\}
$$


is open and dense in a neighbourhood of the origin in $\mathbb{R}^{s}$. Apparently $X$ can be approximated by some $X^{\mu}$ having the property $\mathfrak{B}$, which proves the denseness of this property. Also by the transversality principle, we know that all nearby $s$ parameter families $\tilde{X}^{\mu}$ induce maps $\tilde{F}: M \times \mathbb{R}^{s} \rightarrow N$ for which $\tilde{F} \nwarrow_{M \times\{0\}} S$. From this one can derive openness of the property $\mathfrak{B}$. We conclude these considerations with

3.3. LемMA. Suppose that the above applies for a property $\mathfrak{B}$, in a Whitney $C^{k}$-topology on $\mathfrak{X}^{k}\left(\mathbb{R}^{n}\right), k \geq 1$. Then $\mathfrak{B}$ also is open and dense in $\mathfrak{X}^{\omega}\left(\mathbb{R}^{n}\right)$, using the real analytic topology.

Proof. The openness is clear since the real analytic topology is stronger than the $C^{\infty}$-topology. For the denseness we shall apply lemma 3.2.

So let a real analytic vector field $X$ be given, and a $C^{\infty}$-unfolding $X^{\mu}$ with $X^{0}=X, \mu$ being an $s$-dimensional parameter, such that $X^{\mu}$ has property $\mathfrak{B}$ for $\mu$ open and dense in some neighbourhood of 0 in $\mathbb{R}^{s}$. Now by conclusion (i) of lemma 3.2 we can take a real analytic unfolding $\tilde{X}^{\mu, t}$, where $t>0$ is fixed sufficiently small to ensure that also $\tilde{X}^{\mu, t}$ has the property $\mathfrak{B}$ for $\mu$ open and dense in some neighbourhood of 0 in $\mathbb{R}^{s}$. Next let $\mathcal{U}$ be a neighbourhood of $X$ in the analytic topology. Then by conclusion (ii) of lemma 3.2 we can find $\mu$ with $|\mu|$ sufficiently small to ensure that $\tilde{X}^{\mu, t} \in \mathcal{U}$ and has the property $\mathfrak{B}$.

Remarks. (i) The above considerations also apply for diffeomorphisms, for vector fields and diffeomorphisms subordinate to a volume- or a symplectic structure and for finite parameter families of such dynamical systems.

(ii) The construction above sometimes is modified a little (cf. the proof that generically the stable and unstable manifolds meet transversally). A $C^{k}$-open and -dense property $\mathfrak{B}$ then is translated in terms of transversality as follows: Given is a manifold $N$ with $S \subseteq N$ as before. To each $X$ we associate a compact submanifold $N_{X} \subseteq N$ in a differentiable way and $X$ then has the property $\mathfrak{B}$ if and only if $N_{X}$ 不 $S$. For the rest the procedure remains unchanged.

(d) We conclude this section by generalizing the above to real analytic manifolds, using a fundamental solution of the heat equation. Similar perturbation techniques were used in e.g. Whitney [34], Bochner [4], Morrey [15] and Grauert [10]. For simplicity we first restrict to the orientable case, later on we'll get rid of this assumption.

To be more precise $M$ shall be a real analytic, orientable manifold with a real analytic Riemannian metric. Let $\Delta=d \delta+\delta d$ denote the corresponding LaplaceBeltrami operator, defined on differential forms (for terminology in this part of $\S 3$ cf. e.g. Abraham and Marsden [1]). Then consider the heat equation

$$
\frac{\partial \omega_{t}}{\partial t}=\Delta \omega_{t}
$$

where $\omega_{t}$, for all $t \geq 0$, is a differential form on $M$ of some fixed degree $p$. Due to the Riemannian metric the heat equation for 1-forms carries over to vector fields $\left(X^{b} \leftrightarrow X\right)$. It is known, see e.g. Berger et al. [2] or Cheval [7] that the heat equation 
has a fundamental solution $E_{t}^{p}(x, y)$, meaning that

$$
\omega_{t}(x)=\int_{M} E_{t}^{p}(x, y) \wedge * \omega_{0}(y),
$$

$t \geq 0$. We only have to consider the case where $\omega_{0}$ has compact support. This heat flow is a continuous semigroup, we write $\omega_{t}=e^{-t \Delta} \omega_{0}(t \geq 0)$. One refers to $E_{t}^{p}(x, y)$ as the heat kernel. By Petrovsky's theorem, see Narasimhan [17], in our present case the heat kernel is real analytic, since locally the heat equation is an elliptic partial differential equation with real analytic coefficients. This means that $\omega_{t}$, for $t>0$, will be real analytic (similar for vector fields).

Before carrying over lemma 3.1 to this setting, we first give a few remarks concerning the preservation of a volume- or a symplectic structure. So let $X^{0}$ be a given vector field on $M$ and let $X^{t}(t \geq 0)$ denote its heat evolution (i.e. $\left(X^{t}\right)^{b}=$ $e^{-t \Delta}\left(\left(X^{0}\right)^{b}\right)$. Then we have

3.4. Proposition. Let $\Omega$ be the Riemannian volume form on $M$. Then:

(i) If $\mathscr{L}_{X^{0}} \Omega=0$ then $\mathscr{L}_{X^{\prime}} \Omega=0$ for all $t \geq 0$.

Moreover, if $n=2 m$ and $\tau$ is a symplectic 2-form on $M$ with $\tau \wedge \tau \wedge \cdots \wedge \tau=\Omega$, then:

(ii) If for a function $H^{0}$ one has $\iota_{X}{ }^{0} \tau=d H^{0}$, then $\iota_{X} \tau=d H^{t}$ for all $t \geq 0$, where $H^{t}=e^{-\Delta t} H^{0}$.

Proof (Only of (i), the rest being similar). First note that the heat flow preserves Hodge decomposition, while $e^{-t \Delta} \Omega=\Omega$ for all $t \geq 0$. Now, recalling that $\iota_{Y} \Omega=$ $\pm * Y^{b}$ and that $\mathscr{L}_{Y} \Omega=d\left(\iota_{Y} \Omega\right)$, we conclude that it is sufficient to prove that $d *\left(X^{t}\right)^{b}=0$ for $t>0$, this equality for $t=0$ being given. To this end observe that

$$
\frac{\partial}{\partial t}\left(d *\left(X^{t}\right)^{b}\right)=\Delta\left(d *\left(X^{t}\right)^{b}\right)
$$

implying that

$$
d *\left(X^{t}\right)^{b}=e^{-t \Delta}\left(d *\left(X^{0}\right)^{b}\right)
$$

From this our assertion follows directly.

It remains to establish the perturbation lemma 3.1 for our present setting. For simplicity we restrict to functions ( 0 -forms), but a generalization to $k$-forms is obvious. Our main source is Cheval [7], for more literature we refer to this.

The ingredients we use are

$$
\int_{M} E_{t}(x, y) d y \leq 1 .
$$

(If the metric on $M$ were to be assumed complete with Ricci curvature bounded from below, then here one would have an equality sign, cf. [7, p. 190/191].) Moreover let $x \in M$ be a given point and $\varepsilon$ some positive number, to be chosen suitably small in the end. Assume that on the disc $D_{\varepsilon}(x)$ the Ricci curvatures are bounded from below by $(n-1) \kappa$. Then, for $y \in D_{\varepsilon}(x)$

$$
E_{t}(x, y) \geq(4 \pi t)^{-n / 2} e^{-d^{2}(x, y) / 4 t}\left\{1+R_{\kappa}(d(x, y), t)\right\},
$$

where $d$ denotes the distance and where $R_{\kappa}(r, t)=O\left(r^{2}\right)+O(t)$ as $r^{2}, t \downarrow 0$ locally 
uniform in x. Cf. [7, p. 195]. This is the so called Minakshisundaram-Pleijel formula for Dirichlet kernels. (Such a formula also holds for $k$-forms, cf. [7, p. 337 etc.].)

We first consider part (i) of lemma 3.1. So we have to show that for any $C^{\infty}$-function $\phi$ on $M$, with compact support, $e^{-t \Delta} \phi \rightarrow \phi$ as $t \downarrow 0$, in the weak Whitney $C^{\infty}$-topology. We argue that it is sufficient to prove convergence in the $C^{0}$-topology: Granted $C^{0}$-convergence the $C^{\infty}$-convergence follows by a general argument, using the fact that $\Delta$ commutes with $e^{-t c}$. In fact, for all $k \geq 1$ one has that $\Delta^{k} e^{-t \Delta} \phi \rightarrow \Delta^{k} \phi$ in some Sobolev space, which by Sobolev's lemma (cf. e.g. Narasimhan [17]) gives the desired estimates.

Now, in order to show that $\left(e^{-t \Delta} \phi\right)(x) \rightarrow \phi(x)$, locally uniform in $x$, we have

$$
\begin{aligned}
\left|\phi(x)-\left(e^{-t \Delta} \phi\right)(x)\right| \leq & \max \left\{\left|\phi(x)-\sup _{D_{\varepsilon}(x)} \phi(y) \cdot \int_{D_{\varepsilon}(x)} E_{t}(x, y) d y\right|\right. \\
& \left.\left|\phi(x)-\inf _{D_{\varepsilon}(x)} \phi(y) \cdot \int_{D_{\varepsilon}(x)} E_{t}(x, y) d y\right|\right\} \\
& +|\phi|_{0} \cdot \int_{M-D_{\varepsilon}(x)} E_{t}(x, y) .
\end{aligned}
$$

For $\varepsilon>0$ sufficiently small we estimate by (B)

$$
\int_{D_{\varepsilon}(x)} E_{t}(x, y) d y \leq\left\{1-C\left(t+\varepsilon^{2}\right)\right\}(4 \pi t)^{-n / 2} \int_{D_{\varepsilon}(x)} e^{-d^{2}(x, y) / 4 t} d y,
$$

where $C>0$ is locally uniform in $x$. Also, using (A), we estimate

$$
\int_{M-D_{\varepsilon}(x)} E_{t}(x, y) d y \leq 1-\int_{D_{\varepsilon}(x)} E_{t}(x, y) d y .
$$

Then, as in the proof of lemma 3.1, choose e.g. $\varepsilon \sim t^{\frac{1}{4}}$ and let $t \downarrow 0$.

It remains to show part (ii) of lemma 3.1. Recall that $E_{t}(x, y)$ is real analytic in $x$ and $y$. This implies that for $K \subseteq M$ compact and $\sigma>0$ sufficiently small, we have that $\sup _{x \in K+\sigma} E_{t}(x, y)$ is continuous in $y$. Here $K+\sigma$ is the complex strip of width $\sigma$ around $K$. Then, for all $\phi$ with $\operatorname{supp}(\phi) \subseteq K$ one has

$$
\begin{aligned}
\left|e^{-t \Delta} \phi\right|_{K, \sigma} & =\sup _{K+\sigma}\left|\int_{K} E_{t}(x, y) \phi(y) d y\right| \leq|\phi|_{0} \cdot \sup _{K+\sigma} \int_{K}\left|E_{t}(x, y)\right| d y \\
& \leq|\phi|_{0} \cdot \operatorname{vol}(K) \cdot \sup _{y \in K} \sup _{x \in K+\sigma}\left|E_{t}(x, y)\right| .
\end{aligned}
$$

This estimate appears weaker than (ii) of lemma 3.1, but in fact it is sufficient for the purpose of carrying over lemma 3.3 to the present situation: remember that in each application all bump functions $\phi$ have their supports contained in a fixed compact set $K \subseteq M$.

This finishes our generalization to the case of orientable manifolds. As we said before, the condition that $M$ be orientable can be dropped. In order to see this let $\pi: \tilde{M} \rightarrow M$ be the orientable double cover of $M$. Riemannian metrics and vector fields on $M$ canonically lift to $\tilde{M}$. Also we have an involution $\mathscr{I}: \tilde{M} \rightarrow \tilde{M}$ with $\pi \circ \mathscr{I}=\pi$, which is an isometry for each of these metrics. A vector field $\tilde{X}$ on $\tilde{M}$ projects to a vector field on $M$ if and only if $\mathscr{I}_{*} \tilde{X}=\tilde{X}$. Now we carry out our 
perturbation scheme on $\tilde{M}$. So then we have to make sure that $\mathscr{I}_{*} \tilde{X}=\tilde{X}$ for all our perturbations $\tilde{X}$. This, however, is a direct consequence of the fact that $\mathscr{I}$ is an isometry, which makes it commute with $\Delta$ and therefore with the heat operator $e^{-t \Delta}$.

\section{The Kupka Smale theorems}

In this section we indicate how the various Kupka Smale theorems, compare Kupka [13], Smale [26], Peixoto [19], Robinson [22], Brunovksy [6], Sotomayor [27], Palis and de Melo [18], fit in the scheme of the previous section, so implying that they carry over to the real analytic setting. We shall not exhibit all details here, nor shall we even formulate all the different versions for vector fields, diffeomorphisms or families of these in their various contexts. Instead we refer mostly to the above literature. In fact, to give an idea, we shall only explore to some detail the proof for the general (dissipative) setting for vector fields, as it can be found in Palis and de Melo [18]. After this we shall briefly discuss the Hamiltonian case, compare Robinson [22], in between making some remarks on one-parameter families of general (dissipative) vector fields, see Brunovksy [6], Sotomayor [27].

(a) A proof of theorem 1.1. In Palis and de Melo [18] a $C^{r}$-version $(r \geq 1)$ of theorem 1.1 is proved. We here only need the case where $r=1$. We shall retrace the steps of this $C^{1}$-proof, showing that they are all in accordance with the transversality principle from the previous section.

In the first part of their proof Palis and de Melo [18] show that for any given compact set $D \subseteq M$ it is a $C^{1}$-open and -dense property for vector fields on $\mathbb{R}^{n}$ to have only hyperbolic equilibrum points in $D$. This hyperbolicity can be expressed in terms of transversality as follows. For a given vector field $X$ consider the 1 -jet map $j^{1} X: D \rightarrow T D \times g \ell(n, \mathbb{R})$, defined by $x \rightarrow\left(X(x), D_{x} X\right)$. It is well known that the subset $S \subseteq g \ell(n, \mathbb{R})$, consisting of non-hyperbolic matrices, is (semi) algebraic of codimension larger than 0 . We have that $j^{-1} X$ 不 $D \times\{0\} \times S$ (i.e. with empty intersection), if and only if all singularities of $X$ in $D$ are hyperbolic. Also the perturbation theory of [18] yields a transversal family $X^{\mu}=X+P^{\mu}, P^{0}=0$, where $\mu$ is some $s$-dimensional parameter. Moreover one can ensure that for some compact set $D \subseteq K$ and for all $\mu$ we have $\operatorname{supp}\left(P^{\mu}\right) \subseteq K$. Therefore this property is in accordance with $\S 3$, i.e. a 'Kupka Smale' property.

The second part of the proof from [18] shows that, given our compact set $D \subseteq M$ and given a positive real number $T$, it is a $C^{1}$-open and -dense property for vector fields on $M$, that all their closed orbits, which are contained in $D$ and of period not exceeding $T$, are hyperbolic. In order to fit this property into the scheme of $\S 3$ we choose an arbitrary vector field $X$ in the open and dense set obtained after the first step. In [18] it is then shown that in some suitable $C^{1}$-neighbourhood $\mathscr{U}$ of $X$ the property under consideration is $C^{1}$-open and dense. To be more precise we choose a finite number of disjoint, compact codimension 1 sections $\Sigma_{1}, \Sigma_{2}, \ldots, \Sigma_{L}$ which are all transversal to $X$ and which meet all closed orbits of period not exceeding $T$ in an interior point. The $C^{1}$-neighbourhood $\mathcal{U}$ of $X$ now can be chosen in such a way, that for all $Y \in \mathcal{U}$ this same property holds regarding these fixed sections. Let $\Sigma:=\bigcup_{j=1}^{L} \Sigma_{j}$ and for $Y \in \mathscr{U}$ let $\mathscr{P}_{Y}: \Sigma \rightarrow \Sigma$ be the locally defined Poincaré 
return map of the closed orbits of $Y$. Notice that $Y \rightarrow \mathscr{P}_{Y}$ is differentiable. The closed orbits of $Y$ we have in mind are hyperbolic precisely, then, if the map $\mathscr{P}_{Y}$ has only hyperbolic fixed points. Again this hyperbolicity can be expressed in terms of transversality, the procedure being quite analogous to the one used before. In fact now consider the 1 -jet map $j^{1} \mathscr{P}_{Y}: \Sigma \rightarrow \Sigma \times \Sigma \times \operatorname{Gl}(n-1, \mathbb{R})$, defined by $x \rightarrow$ $\left(x, \mathscr{P}_{Y}(x), D_{x} \mathscr{P}_{Y}\right)$ and make the following adjustments: Instead of the zero section $D \times\{0\} \subseteq T D$ here take the diagonal $\Delta \subseteq \Sigma \times \Sigma$ and replace the set $S \subseteq g \ell(n, \mathbb{R})$ by a similar set in $\operatorname{Gl}(n-1, \mathbb{R})$. Also in this case the perturbation theory of [18] gives a transversal family $Y^{\mu}=Y+P^{\mu}, P^{0}=0$, for some $s$-dimensional parameter and again one can arrange that for all $\mu$ we have $\operatorname{supp}\left(P^{\mu}\right) \subseteq K$, for some compact set $D \subseteq K$. So once more we are completely in the setting of $\S 3$.

In the third and last part of their proof Palis and de Melo [18] deal with the transversality of the stable and unstable manifolds of the critical elements. We proceed as before. Having chosen $D$ and $T$ we fix an arbitrary vector field $X$ in the $C^{1}$-open and -dense set obtained at this moment. So $X$ in $D$ has finitely many critical elements $\sigma_{1}, \sigma_{2}, \ldots, \sigma_{L}$, the period of which do not exceed $T$. All these critical elements are hyperbolic. For each $1 \leq j \leq L$ we take compact neighbourhoods $W_{0}^{s}\left(\sigma_{j}\right)$ and $W_{0}^{u}\left(\sigma_{j}\right)$ in the stable, resp. unstable, manifolds $W^{s}\left(\sigma_{j}\right)$ and $W^{u}\left(\sigma_{j}\right)$, such that the boundaries $\partial W_{0}^{s}\left(\sigma_{j}\right)$ and $\partial W_{0}^{u}\left(\sigma_{j}\right)$ are fundamental domains for the corresponding invariant manifolds. Let $\Sigma_{j}^{s}$ be a codimension 1 submanifold of $M$ that is transversal to $X$ and to the local stable manifold of $\sigma_{j}$, which it meets in $\partial W_{0}^{s}\left(\sigma_{j}\right)$. Now for a sufficiently small $C^{1}$-neighbourhood $U$ of $X$ one has that if $Y \in \mathcal{U}$, then $Y$ is transversal to each $\Sigma_{j}^{s}$, while the critical elements of $Y$ of period not exceeding $T$ are hyperbolic and near the corresponding critical elements of $X$. Thus, if $\sigma_{1}(Y), \ldots, \sigma_{L}(Y)$ are these critical elements, then there exist compact neighbourhoods $W_{0}^{s}\left(\sigma_{j}, Y\right)$ of $\sigma_{j}(Y)$ in $W^{s}\left(\sigma_{j}, Y\right)$ with boundary the intersection of $\Sigma_{j}^{s}$ with $W_{0}^{s}\left(\sigma_{j}, Y\right)$. Note that the map $Y \rightarrow W_{0}^{s}\left(\sigma_{j}, Y\right)$ is continuous regarding the $C^{1}$-topologies. Similarly one finds compact neighbourhoods $W_{0}^{u}\left(\sigma_{j}, Y\right)$ of $\sigma_{j}(Y)$ in $W^{u}\left(\sigma_{j}, Y\right)$ with corresponding continuity. Then, still following [18], for each positive integer $m$ we define $W_{m}^{s}\left(\sigma_{j}, Y\right):=Y_{-m}\left(W_{0}^{s}\left(\sigma_{j}, Y\right)\right)$ and $W_{m}^{u}\left(\sigma_{j}, Y\right):=$ $Y_{m}\left(W_{0}^{u}\left(\sigma_{j}, Y\right)\right)$ where as before $Y_{t}$ denotes the time $t$ evolution of the vector field $Y$. Observe that $W_{m}^{s}\left(\sigma_{j}, Y\right)$ and $W_{m}^{u}\left(\sigma_{j}, Y\right)$ are compact manifolds with boundary, varying $C^{1}$-continuously as $Y$ varies over $U$. Also observe that $W^{s}\left(\sigma_{j}, Y\right)=$ $\bigcup_{m \geq 0} W_{m}^{s}\left(\sigma_{j}, Y\right)$ and $W^{u}\left(\sigma_{j}, Y\right)=\bigcup_{m \geq 0} W_{m}^{u}\left(\sigma_{j}, Y\right)$. Now for fixed $m$ we consider the set of $Y \in \mathcal{U}$ for which $W_{m}^{s}\left(\sigma_{j}, Y\right)$ is transversal to $W_{m}^{u}\left(\sigma_{k}, Y\right)$, for all $j$ and $k$, proving that this set is $C^{1}$-open and -dense in $\mathcal{U}$. In fact it is sufficient to do this for a fixed pair of $j$ and $k$. For a given choice of $m, j$ and $k$ we shall fit this transversality property in the terms of $\S 3$. To this end consider the compact set $K:=W_{m}^{u}\left(\sigma_{j}, Y\right) \cap W_{m}^{s}\left(\sigma_{j}, Y\right)$. Palis and de Melo [18] cover $K$ by a finite number of flowboxes $\phi_{Y, l}: A_{l} \rightarrow\left[-b_{l}, b_{l}\right] \times I^{n-1}(1 \leq l \leq M)$ where, if $t$ is the first coordinate of the product $\left[-b_{l}, b_{l}\right] \times I^{n-1}$, then $\phi_{Y, l}$ conjugates $Y$ on the set $A_{l}$ to the standard vector field $\partial / \partial t$. Again $\phi_{Y, l}$ depends continuously on $Y$, regarding $C^{1}$-topologies. Observe that it is no restriction to assume that for each index $l$ the set

$$
\left\{b_{l}\right\} \times I^{n-1} \cap \phi_{Y, l}\left(A_{l} \cap W_{m}^{s}\left(\sigma_{k}, Y\right)\right)
$$


is independent of $Y \in \mathcal{U}$. In fact we may let it correspond to $S_{l} \subseteq I^{n-1}$ which is, for example, parallel to one of the coordinate 'planes'. Carrying on for a fixed index $l$, which as before is no real restriction, we now formulate as our transversality condition

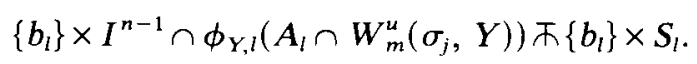

Compare remark (ii) of lemma 3.3 The rest of the argument runs as before. The perturbation theory of $[\mathbf{1 8}]$ provides a transversal family $Y^{\mu}=Y+P^{\mu}, P^{0}=0$, for some $s$-dimensional parameter. In this case one has that $\operatorname{supp}\left(P^{\mu}\right) \subseteq A_{l}$ for all $\mu$. Again we see that the considered property is in accordance with $\S 3$. Up to this point, $D, T, L, m, j, k$ and $l$ being fixed, we can carry over the above proof to our analytic setting. Our proof of theorem 1.1 then is completed in the usual way, taking countably many intersections. Also compare $\S 3$.

(b) We continue with a few remarks on the general (dissipative) case with a parameter. Brunovksy [6] and Sotomayor [27] prove a Kupka Smale theorem for 1-parameter families of vector fields and diffeomorphisms, working in the class $C^{r}$, $r \geq 5$. Although these results have been formulated for a compact manifold, they can easily be modified for the present case. Obviously when a parameter is present mild violations of both properties in theorem 1.1 generically cannot be avoided in a countable set of parameter points. In these points codimension 1 bifurcations occur, a list of which can be found in [6], [27]. For our purposes it is important that they can all be described in terms of the transversality principle of $\S 3$, now formulated for 1-parameter families of dynamical systems on $M$, or rather for so called 'vertical' systems on $M \times \mathbb{R}$. Again the perturbation theory of [6], [27], together with the scheme of $\S 3$, yields that the results carry over to the real analytic setting.

(c) On the Hamiltonian case. We refer to Robinson [22] who proves a 'complete' list of differentiable Kupka Smale theorems for volume preserving dynamical systems, for symplectic diffeomorphisms and 1-parameter families of these, as well as for Hamiltonian vector fields. Notice that for Hamiltonian systems the energy plays the role of parameter, see above. We claim that by our methods all these results carry over to the real analytic case.

We formulated theorem 1.2 as an example of this. For completeness sake we give a brief description of the properties $\mathrm{H}-2$ and $\mathrm{H}-3$. A Hamiltonian vector field is said to have the property $\mathrm{H}-2$ if:

(i) all equilibrium points are elementary;

(ii) all closed orbits lie on 1-parameter families of closed orbits;

(iii) most closed orbits are elementary (not all of them generically can be, compare part (b) of this section).

The invariant manifolds of the critical elements that we consider again here are the (strong) stable and unstable manifolds. In $\S 2$ we already observed that the invariant manifolds of the closed orbits are parametrized by the energy. We recall that for an analytic Hamiltonian system these invariant manifolds are analytic, while near a 1-elementary closed orbit the invariant manifolds of the orbit cylinder also depend analytically on the energy. Now a Hamiltonian system has property $\mathrm{H}-3$ if: 
(i) all equilibria lie on different energy levels;

(ii) the stable manifold of an equilibrium meets its own unstable manifold transversally in the energy level;

(iii) the stable (resp. unstable) manifold of an equilibrium point meets all unstable (resp. stable) manifolds of closed orbits transversally in the energy level;

(iv) all the stable and unstable manifolds of families of 1-elementary closed orbits meet transversally (not necessarily transversally in all energy levels, compare part (b) of this section);

(v) the stable and unstable manifolds for all but a countable number of closed orbits meet transversally within the energy level;

(vi) these countably many closed orbits all are 1-elementary.

Again one can show that all these properties and Robinson's $C^{2}$-perturbation techniques [22] fit in the scheme of $\S 3$, also compare Robinson [23], so implying theorem 1.2.

\section{REFERENCES}

[1] R. Abraham \& J. E. Marsden. Foundations of Mechanics. Benjamin-Cummings, 1978.

[2] M. Berger, P. Gauduchon \& E. Mazet. Le Spectre d'une Variété Riemannienne. Springer-Verlag, Lecture Notes in Maths 194, 1971.

[3] P. Billingsley. Probability and Measure. Wiley and Sons, 1979.

[4] S. Bochner. Analytic mapping of compact Riemann spaces into Euclidean space. Duke Math. J. 3 (1937), 339-354.

[5] H. W. Broer \& G. Vegter. Subordinate Sil'nikov bifurcations near some singularities of vector fields having low codimensions. Ergod. Th. \& Dynam. Sys. 4 (1984), 509-525.

[6] P. Brunovksy. On one parameter families of diffeomorphisms. Commentationes Mathematicae Universitatis Carolinae 11 (1970), 559-582.

[7] I. Cheval. Eigenvalues in Riemannian Geometry. Academic Press, 1984.

[8] S-N. Chow \& J. K. Hale. Methods of Bifurcation Theory. Springer-Verlag, 1982.

[9] E. A. Coddington \& N. Levinson. Theory of Ordinary Differential Equations. McGraw-Hill, 1955.

[10] H. Grauert. On Levi's problem and the imbedding or real analytic manifolds. Ann. Math. 68, (1958), $460-472$.

[11] P. Hartman. Ordinary Differential Equations. Wiley and Sons, 1964.

[12] M. W. Hirsch. Differential Topology. Springer Verlag, Graduate Texts in Maths 33, 1976.

[13] I. Kupka. Contribution à la théorie des champs génériques. Contrib. Diff. Eqs. 2 (1963), 457-484; ibid 3 (1964), 411-420.

[14] J. Leslie. On the group of real analytic diffeomorphisms of a compact real analytic manifold. Trans. Am. Math. Soc. 274, (1982), 651-669.

[15] C. B. Morrey. The analytic embedding of abstract real-analytic manifolds. Ann. Math. 68 (1958), 159-201.

[16] J. K. Moser. Non-existence of integrals for canonical systems of differential equations. Comm. Pure Appl. Math. 8 (1955), 409-436.

[17] R. Narasimhan. Analysis of Real and Complex Manifolds. North-Holland, 1968.

[18] J. Palis \& W. de Melo. Geometric Theory of Dynamical Systems. Springer-Verlag, 1982.

[19] M. M. Peixoto. On an approximation theorem of Kupka and Smale. J. Diff. Eq. 3 (1966), 214-227.

[20] C. C. Pugh. The closing lemma. Amer. J. Math. 89 (1967), 956-1009.

[21] C. C. Pugh. An improved closing lemma and a general density theorem, Amer. J. Math. 89 (1967), 1010-1021.

[22] R. C. Robinson. Generic properties of conservative systems I, II, Amer. J. Math. 92 (1970), 562-603; 897-906.

[23] R. C. Robinson. Generic one parameter families of symplectic matrices. Amer. J. Math. 93 (1971), 116-122.

[24] L. Schwartz. Séminaire Schwartz. 2ème année, 1954-1955. 
[25] C. L. Siegel. Ueber die Existenz einer Normalform analytischer Hamiltonscher Differentia]gleichungen in der Nähe einer Gleichgewichtslösung. Math. Ann. 128 (1954), 144-170.

[26] S. Smale. Stable manifolds for differential equations and diffeomorphisms. Ann. Scuola Normale Superiore Pisa 18 (1963), 97-116.

[27] J. Sotomayor. Generic bifurcations of dynamical systems. In: Dynamical Systems (ed. M. M. Peixoto), Academic Press 1973.

[28] S. J. Van Strien. Center manifolds are not $C^{\infty}$. Math. Z. 116 (1979), 143-145.

[29] F. Takens. Hamiltonian systems: generic properties of closed orbits and local perturbations. Math. Ann. 188 (1970), 304-312.

[30] F. Takens. Singularities of vector fields. Publ. I.H.E.S. 43 (1974), 47-100.

[31] F. Takens. A non-stabilizable jet of a singularity of a vector field; the analytic case. In: Algebraic and Differential Topology-Global Differential Geometry (ed. G. M. Rassias), Teubner Verlag, 1984.

[32] F. Takens. Measure and category. Preprint, University of Groningen, 1985.

[33] K. Weierstrass. Ueber die analytische Darstellbarkeit sogenannter willkürlicher Funktionen einer reellen Veränderlichen. Sitzungsberichte der Akademie zu Berlin (1885), 633-639; 789-805.

[34] H. Whitney. Analytic extensions of differentiable functions defined in closed sets. Trans. Amer. Math. Soc. 36 (1934), 63-89.

[35] E. Zehnder. Homoclinic points near elliptic fixed points. Comm. Pure Appl. Math. 26 (1973), 131-182. 